\title{
Avaliação de conteúdos educativos do YouTube® sobre prevenção da cárie dentária
}

\author{
Analysis of YouTube ${ }^{\circledR}$ educational videos on prevention of dental caries \\ Evaluación de contenido educativo en YouTube® sobre prevención de caries
}

Gabriel Quirino Dalpoz

ORCID: https://orcid.org/0000-0002-3873-9375 Universidade Estadual de Maringá, Brasil E-mail: gabrieldalpoz73@gmail.com

Maura Sassahara Higasi

ORCID: https://orcid.org/0000-0001-5639-5193 Universidade Estadual de Londrina, Brasil E-mail:maurash@uel.br

Tânia Harumi Uchida

ORCID: https://orcid.org/0000-0001-8170-1092 Universidade Estadual de Maringá, Brasil E-mail: taniaharumi@gmail.com

Mitsue Fujimaki

ORCID: https://orcid.org/0000-0002-7824-3868 Universidade Estadual de Maringá, Brasil E-mail: mfujimaki@gmail.com

\begin{abstract}
Resumo
Este estudo teve como objetivo avaliar a qualidade e a confiabilidade de vídeos de animação disponíveis no YouTube ${ }^{\circledR}$ sobre a prevenção da cárie dentária ao público infantil. Inicialmente, foi realizada uma busca no YouTube ${ }^{\circledR}$ utilizando expressões relacionadas ao tema: "Como cuidar dos dentes", "Como escovar os dentes animação", "Prevenção de cárie desenho", "Como escovar os dentes criança", "Como escovar os dentes desenho" e "Como escovar os dentes infantil". Foram encontrados 132 vídeos, dos quais 84 não foram incluídos, por atenderem aos critérios de exclusão. Foram avaliados a qualidade e confiabilidade de 48 vídeos, por meio dos instrumentos DISCERN adaptado e JAMA. Os vídeos tiveram uma somatória de 165.459.240 visualizações no dia da coleta $(\bar{X}=3.447 .068)$. Os vídeos foram considerados de qualidade variável pelo DISCERN ( $\bar{X}=29,5$; variando de 14 a 44$)$ e de baixa confiabilidade pelo JAMA ( $\overline{\mathbf{X}}=0,89$; variando de 0,25 a 2). Conclui-se que os vídeos de animação de curta duração disponíveis no YouTube ${ }^{\circledR}$ sobre prevenção de cárie dentária para o público infantil, analisados neste estudo, foram considerados de qualidade variável e de baixa confiabilidade, necessitando de melhorias para promover uma orientação mais adequada ao grande público que os assiste.
\end{abstract}

Palavras-chave: Saúde bucal; Internet; Odontologia.

\begin{abstract}
This study intended to evaluate both quality and reliability of short animated videos about dental caries prevention, published on YouTube ${ }^{\circledR}$ and directed to children. The first step was running a search with expressions related to the topic: "How to care for teeth", "How to brush teeth animation", "Drawing prevention of tooth decay", "How to brush a child's teeth", "How to brush teeth design" and "How to brush children's teeth". 132 videos were found, of which 84 were excluded by the exclusion criteria. The quality and reliability of the 48 videos were evaluated using the adapted DISCERN and JAMA instruments. The videos had a total of 165.459 .240 views on the day of collection $(\bar{x}=3.447 .068$ ), were considered of variable quality by DISCERN ( $\bar{x}=29.5$; ranging from 14 to 44 ) and of low reliability by JAMA ( $\bar{x}=0.89$; ranging from 0.25 to 2 ). It is concluded that the short animated videos for children on YouTube ${ }^{\circledR}$ about dental caries prevention, included in this study, were considered of variable quality and of low reliability, needing improvements to promote a more adequate orientation to the general public that watch them.
\end{abstract}

Keywords: Oral health; Internet; Dentistry.

\section{Resumen}

Este estudio tuvo como objetivo evaluar la calidad y confiabilidad de los videos animados disponibles en YouTube ${ }^{\circledR}$ sobre la prevención de la caries dental en los niños. Inicialmente se realizó una búsqueda en YouTube® utilizando expresiones relacionadas con el tema: "Cómo cuidar los dientes", "Animación de cómo cepillarse los dientes", "Prevención de caries dibujando", "Cómo cepillarse los dientes para un niño", "Diseño de Cómo cepillarse los dientes" y "Cómo cepillar los dientes de los niños". Se encontraron 132 videos, de los cuales 84 fueron excluidos por los criterios de exclusión. La calidad y confiabilidad de los 48 videos se evaluaron utilizando los instrumentos 
DISCERN adaptado y JAMA. Los videos tuvieron una suma de 165.459.240 visualizaciones el día de la recolección $(\overline{\mathrm{X}}=3.447 .068)$. Los videos fueron considerados de calidad variable por DISCERN ( $\overline{\mathrm{X}}=29.5$; rango de 14 a 44$)$ y de baja confiabilidad por JAMA ( $\bar{X}=0.89$; rango de 0.25 a 2). Se concluye que los videos cortos de animación disponibles en YouTube® sobre la prevención de la caries dental infantil, analizados en este estudio, fueron considerados de calidad variable y baja confiabilidad, necesitando mejoras para promover una orientación más adecuada al público en general.

Palabras clave: Salud bucal; Internet; Odontología.

\section{Introdução}

O período pandêmico provocado pelo novo coronavírus fez com que grande parte da população brasileira permanecesse em isolamento social com o intuito de conter o avanço do vírus (Deslandes \& Coutinho, 2020). O Ministério da Educação, por meio da Portaria n 544 de 16 de junho de 2020, autorizou a substituição das aulas presenciais por aulas em meios digitais enquanto durar este período de pandemia da COVID-19. Esta portaria permitia que as Instituições de Ensino de nível fundamental, médio e superior trabalhassem por meio de plataformas de ensino para realização das atividades de forma remota e uma dessas plataformas é a do YouTube ${ }^{\circledR}$. Dessa forma, na área da educação verificou-se a abertura de novas possibilidades para a aprendizagem, para a incorporação de conceitos, obtenção de saberes, favorecendo a melhoria das oportunidades para o indivíduo, além de poder proporcionar autonomia, visão crítica, percepção de valores e conhecimentos e empoderamento (Santos, et al., 2012).

$\mathrm{Na}$ área da saúde bucal, as medidas educativas buscam conscientizar os indivíduos para os cuidados necessários para a manutenção da saúde, de modo a estimular a autonomia e a valorização do autocuidado, para evitar o aparecimento da doença, pois o tratamento especializado é de alto custo, menos acessível a grande parte da população, sendo preferível prevenir a doença e suas consequências (Dinelli, et al., 2000; Santos, et al., 2003; Petersen, 2003).

Com o intuito de facilitar o processo educativo, novas estratégias estão sendo incorporadas, permitindo a utilização de outros meios de comunicação e de divulgação. Com a globalização, nesta era digital, de transformações sociais, de acesso às informações, a internet tornou-se um veículo de transmissão e de busca por materiais educativos, referências bibliográficas, contato de pessoas, arquivos de domínio público, listas de discussão, entre outros (Henning, 2012; Aranha \& Miranda, 2019). Segundo dados da Pesquisa Brasileira de Mídia, metade dos brasileiros utilizam a internet com regularidade para se informar, se divertir e estudar. Uma das plataformas mais acessadas no Brasil e no mundo é o YouTube ${ }^{\circledR}$, uma plataforma aberta de compartilhamento de vídeos acessível e muito utilizada (Rodrigues, 2008; Moura \& Freitas, 2018), sendo os vídeos de conteúdos educacionais entre os mais procurados (Burke; Snyder, 2008). Apesar do YouTube ${ }^{\circledR}$ não ter sido criado exclusivamente com a finalidade educacional, ele vem ganhando cada vez mais espaço nesse meio. Muitas pessoas leigas, professores, escolas e Instituições de Ensino Superior (IES) têm feito o compartilhamento de vídeos de caráter educativo em canais individuais ou institucionais do YouTube ${ }^{\circledR}$ (Snelson, 2011; Rodrigues, et al., 2017). Na área da saúde, a utilização do YouTube ${ }^{\circledR}$ para divulgação de conteúdos educativos tem sido observada, demonstrando o potencial da disseminação desta tecnologia educacional à população em geral (Silva; Blumentritt; Cordeiro, 2021).

Entretanto, as informações presentes nos vídeos do YouTube ${ }^{\circledR}$ têm sido divulgadas sem uma análise crítica sistemática sobre seu conteúdo, visto que os usuários que têm conta no YouTube® são responsáveis pelas informações dos vídeos que postam. Tanto a abertura de uma conta, quanto a postagem de vídeos são procedimentos simples (Moura \& Freitas, 2018). Assim, os internautas devem se atentar à veracidade do que se lê ou assiste na internet (Monteiro, 2001).

De acordo com o Conselho Regional de Medicina do Estado de São Paulo (2019), todas as informações relacionadas à saúde disponíveis na internet deveriam ser precisas, atualizadas e de fácil compreensão, com uma linguagem objetiva e fundamentada cientificamente. Todavia, considerando o grande número de vídeos sobre saúde disponível na internet, muitos podem apresentar conteúdos adequados ou não, e não ter sido desenvolvidos por uma equipe qualificada, sendo comum 
pessoas influentes do meio artístico ou das redes sociais, indicarem a utilização de produtos sem comprovação científica para prevenção de doenças, como por exemplo, a cárie dentária (Fujimaki, 2018). Esta é uma realidade vivenciada na era digital, que tem levado pessoas leigas a acreditarem em informações divulgadas por Youtubers que não têm o conhecimento necessário sobre a temática divulgada.

O uso da internet e a incorporação de tecnologias de informação e comunicação nas ações de educação em saúde têm contribuído para a produção e divulgação de conhecimentos, a fim de transformar a utilização empírica em uma abordagem científica (Aguiar \& Cassiani, 2007). A utilização de vídeos educativos para a saúde pode permitir o aprofundamento de diferentes temas, bem como a melhor compreensão das informações, uma vez que a forma e o conteúdo de um vídeo podem despertar a curiosidade e o interesse, estimulando o indivíduo para o autocuidado e a promoção da saúde (Boctor, 2013; De Moraes Pinto, et al., 2015).

Dessa forma, o objetivo deste estudo foi avaliar a qualidade e a confiabilidade de vídeos de animação disponíveis no YouTube® sobre a prevenção da cárie dentária ao público infantil.

\section{Metodologia}

\subsection{Delineamento da Pesquisa}

Trata-se de um estudo do tipo exploratório, com abordagem quantitativa e qualitativa (Creswell \& Clark, 2013), realizado a partir da coleta de dados no sítio de compartilhamento de vídeos YouTube ${ }^{\circledR}$ (endereço eletrônico: www.youtube.com). Foi realizada inicialmente uma busca no YouTube ${ }^{\circledR}$ utilizando-se os termos: "Como cuidar dos dentes", "Como escovar os dentes animação", "Prevenção de cárie desenho", "Como escovar os dentes criança", "Como escovar os dentes desenho" e "Como escovar os dentes infantil". Foram coletados os seguintes dados dos vídeos: número de visualizações, likes, dislikes, comentários e inscritos nos canais de postagem na data da coleta (4 de dezembro de 2019), dentre outros dados referentes ao conteúdo dos vídeos e fontes utilizadas.

\subsection{Critérios de Elegibilidade}

Foram definidos como critérios de inclusão: vídeos de curta duração de até 4 minutos, vídeos de animação (desenhos animados) e vídeos disponíveis na data da coleta. Os critérios de exclusão foram: vídeos em idiomas estrangeiros, vídeos cujo conteúdo não estava relacionado à prevenção da cárie dentária e vídeos repetidos.

\subsection{Análise dos Dados}

Primeiramente foi realizada uma análise piloto para formação e consenso dos avaliadores sobre os dados a serem coletados e os critérios para a avaliação dos vídeos. A partir da definição das variáveis da pesquisa, dois avaliadores analisaram separadamente todos os vídeos, e em situações de divergência das análises, um terceiro avaliador auxiliou no consenso. Foram analisadas as temáticas principais e conteúdo dos vídeos.

Para a análise dos vídeos foram utilizados dois instrumentos de avaliação: 1) DISCERN (instrumento que avalia a confiabilidade, qualidade geral e qualidade das informações), palavra inglesa que significa "discernir”, e 2) Confiabilidade a partir do instrumento do Journal of the American Medical Association (JAMA). As questões do DISCERN foram adaptadas para a análise em questão, totalizando 10 questões (Quadro 1). 
Quadro 1: Instrumento de avaliação de confiabilidade, qualidade geral e qualidade das informações (DISCERN) adaptado.

\begin{tabular}{|l|l|}
\hline 1. & Os objetivos são claros? \\
\hline 2. & Alcança seus objetivos? \\
\hline 3. & É relevante? \\
\hline 4. & É claro que fontes de informação foram usadas para compilar a publicação (além do autor ou produtor)? \\
\hline 5. & É equilibrado e imparcial? (em relação à alguma empresa). \\
\hline 6. & Descreve como funciona cada procedimento de prevenção? \\
\hline 7. & Descreve os benefícios de cada procedimento de prevenção? \\
\hline 8. & Descreve o que aconteceria se nenhum procedimento de prevenção fosse usado? \\
\hline 9. & Descreve como as opções de tratamento afetam a qualidade de vida geral? \\
\hline 10. & Fornece suporte para a tomada de decisão compartilhada (país)? \\
\hline
\end{tabular}

Fonte: Autores.

As perguntas 1 a 5 abordaram a confiabilidade da publicação e ajudam a considerar se ela pode ser confiável como fonte de informações sobre as opções de tratamento. As perguntas 6 a 10 concentraram-se em detalhes específicos das informações sobre as opções de tratamento. As perguntas se relacionavam à escolha ou às opções de tratamento abordadas, e não a todas as opções de tratamento existentes. A pergunta 6 referia-se aos tratamentos 'ativos' descritos na publicação, podendo incluir o autocuidado. As opções 'sem tratamento' são tratadas separadamente na pergunta 8. Cada pergunta foi classificada em uma escala de 5 pontos, variando de Não a Sim. A escala de classificação foi projetada para ajudar a decidir se o critério de qualidade em questão foi ou não cumprido pela publicação. Os critérios utilizados foram: nota 5 foi dada se a resposta à pergunta fosse um "sim" definitivo - o critério de qualidade foi completamente preenchido; parcialmente (2-4) foi fornecido quando a publicação considerada não atendia ao critério em sua totalidade; 1 foi dado se a resposta à pergunta foi um "não" definitivo - o critério de qualidade não foi cumprido. A partir da somatória dos pontos de cada item foi obtida uma nota entre 10 (qualidade ruim) e 50 (ótima qualidade) (Charnock, 1998).

A confiabilidade dos vídeos foi avaliada utilizando os critérios do JAMA. Cada vídeo recebeu uma pontuação de 0 a 4; a pontuação média do JAMA de 3 ou acima é sugerida como de alta qualidade. Nesses critérios foram avaliadas as seguintes informações: autoria (identificação dos autores/colaboradores); atribuição (lista fontes de informação ou referências); divulgação (declaração de propriedade, publicidade, conflito de interesses); moeda (indica a data em que o conteúdo foi publicado ou atualizado). Recebendo as demais notas: 0 (quando a informação estava ausente); 1 (se apenas citava a informação na descrição do vídeo ou no próprio vídeo); 2 (se além de ter a informação na descrição mostrava a logo/autoria/fonte de informação/data no vídeo); 3 (quando a informação era citada pelos personagens no vídeo); 4 (além de citado, no vídeo e descrição, ela era detalhada). A média destas notas dos 4 itens revelou a confiabilidade do vídeo, que pode ser de 0 (baixa confiabilidade) e 4 (alta confiabilidade) (Silberg, et al., 1997).

Os dados referentes aos números de visualizações, likes, dislikes, comentários e tempo de postagem dos vídeos foram analisados por meio da Correlação de Pearson, utilizando o software Microsoft Excel® 16.0.

\section{Resultados}

Foram selecionados um total de 132 vídeos e após a verificação dos critérios de elegibilidade, foram submetidos à análise 48 vídeos. 
Figura 1: Fluxograma do processo de seleção dos vídeos do YouTube®.

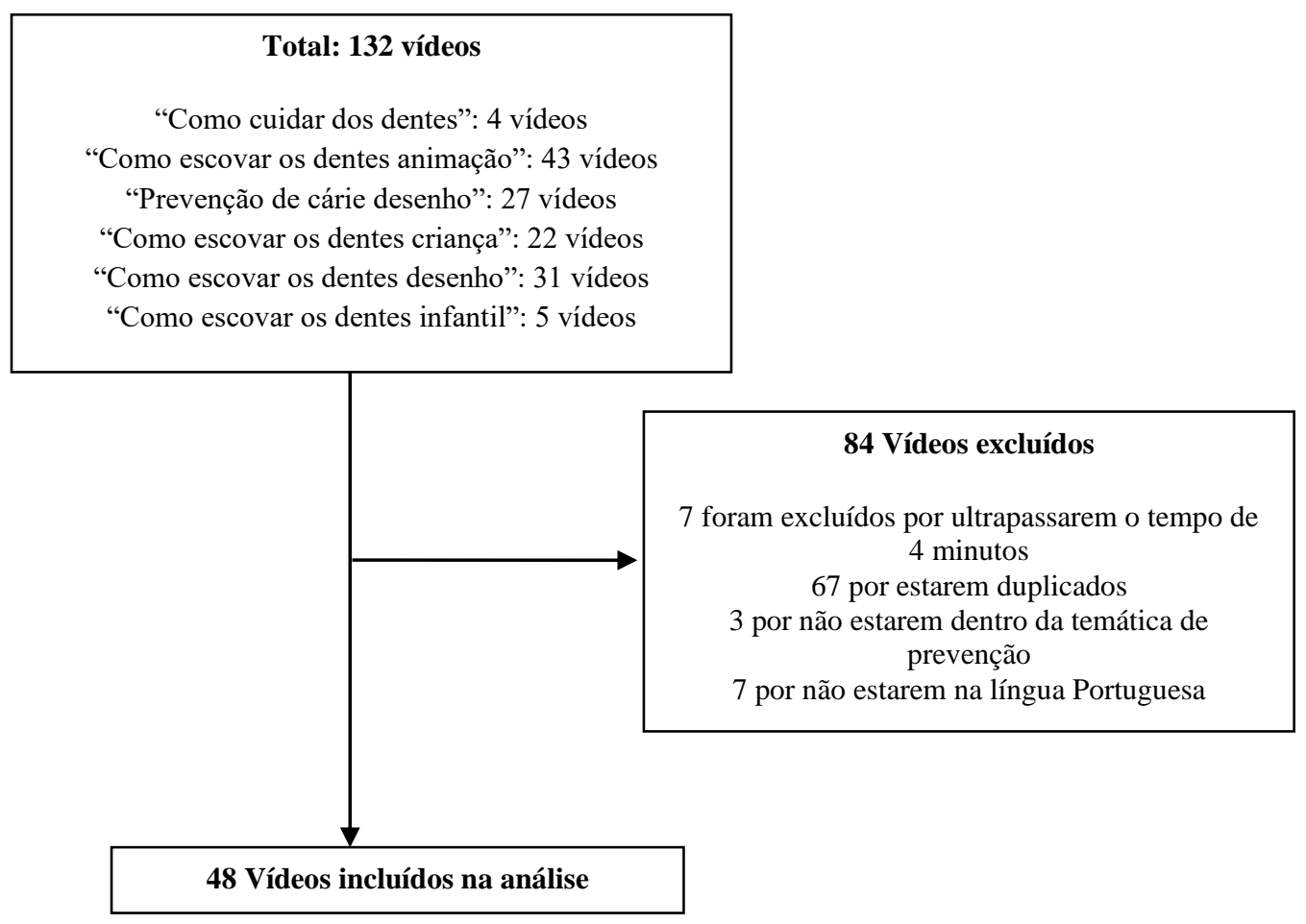

Fonte: Autores.

A Tabela 1 apresenta uma síntese dos dados dos vídeos coletados no sítio do YouTube®.

Tabela 1: Variação do número de inscritos nos canais do YouTube®, likes, dislikes, comentários, tempo de postagem dos vídeos avaliados, classificados de acordo com a faixa de visualizações $(n=48)$. Valores referentes à data da coleta $(4$ de dezembro de 2020).

\begin{tabular}{lcccccc}
\hline \multicolumn{1}{c}{ Visualizações } & $\begin{array}{c}\text { Número de } \\
\text { vídeos }\end{array}$ & Inscritos no canal & Likes & Dislikes & Comentários & Tempo de postagem \\
\hline 0 a 9.999 & 15 & 0 a 333.000 & 2 a 157 & 0 a 17 & 0 a 22 & 6 meses a 9 anos \\
10.000 a 99.999 & 11 & 74 a 5.560 .000 & 20 a 435 & 0 a 203 & 0 a 27 & 5 meses a 11 anos \\
100.000 a 999.999 & 10 & 4.340 a 3.591 .000 & 0 a 1.400 & 0 a 1.400 & 2 a 222 & 4 meses a 8 anos \\
1.000 .000 a & 12 & 4.260 a 5.300 .000 & 0 a 124.000 & 0 a 124.000 & 3 a 2.760 & 8 meses a 11 anos \\
80.000 .000 & & & & & & \\
\hline
\end{tabular}

Fonte: Próprios autores.

Verificou-se que os vídeos tinham uma somatória de 165.459.240 visualizações no dia da coleta ( $\overline{\mathrm{X}}=3.447 .068)$; os 14 vídeos com mais likes estavam entre os 15 mais visualizados; o vídeo mais visualizado tinha 77.983 .751 visualizações, ele também era o mais comentado ( 2.760 comentários); a média de visualizações foi de 3.447.068 e mediana de 72.957; a mediana dos comentários foi de 5 e a média de 125 .

A Tabela 2 apresenta os coeficientes de correlação de Pearson entre variáveis coletadas sobre os vídeos no site do YouTube . Verificou-se que existe uma forte correlação positiva do número de visualizações, likes, dislikes e comentários entre si, mostrando que os vídeos mais visualizados tinham um maior número de interações. Além disso, foi possível verificar uma fraca correlação entre inscritos, tempo de postagem e demais variáveis. 
Research, Society and Development, v. 11, n. 1, e26011124693, 2022

(CC BY 4.0) | ISSN 2525-3409 | DOI: http://dx.doi.org/10.33448/rsd-v11i1.24693

Tabela 2: Coeficiente de correlação do número de visualizações, quantidade de likes, dislikes, comentários, inscritos e data de postagem entre si.

\begin{tabular}{ccccccc}
\hline Variáveis & Visualizações & Likes & Dislikes & Comentários & Inscritos & Data de postagem \\
\hline Visualizações & 1 & 0,96 & 0,97 & 0,97 & 0,31 & $-0,04$ \\
Likes & 0,96 & 1 & 0,98 & 0,95 & 0,33 & $-0,07$ \\
Dislikes & 0,97 & 0,98 & 1 & 0,93 & 0,39 & $-0,1$ \\
Comentários & 0,97 & 0,95 & 0,93 & 1 & 0,2 & 0,01 \\
Inscritos & 0,31 & 0,33 & 0,39 & 0,2 & 1 & $-0,33$ \\
Data de postagem & $-0,04$ & $-0,07$ & $-0,1$ & 0,01 & $-0,33$ & 1 \\
\hline
\end{tabular}

Fonte: Próprios autores.

A Figura 2 apresenta as temáticas abordadas nos vídeos analisados. Verificou-se que a importância da higiene bucal e a frequência da escovação foram os conteúdos mais frequentes.

Figura 2: Temáticas abordadas nos vídeos analisados $(\mathrm{N}=48)$.

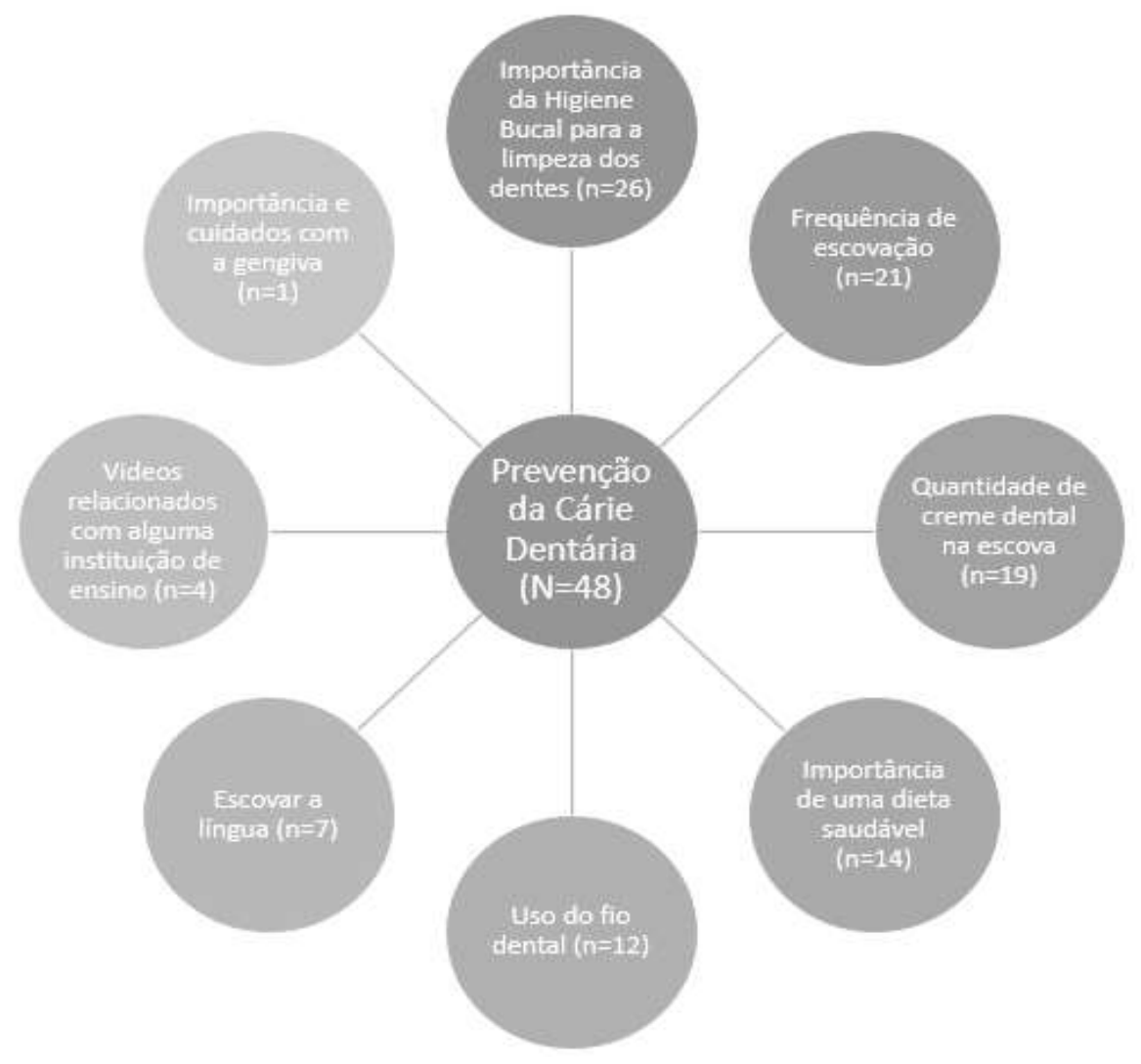

Fonte: Próprios autores.

Em relação à fonte das informações apresentadas no vídeo, apenas um mencionava a referência e os outros 47 vídeos não apresentaram nenhuma referência ou fonte das informações. Do total, 15 vídeos trouxeram a informação inadequada da quantidade de creme dental que deve ser utilizada.

Os resultados da avaliação da qualidade e confiabilidade dos vídeos estão apresentados na Tabela 3. 
Tabela 3: Valores da média, mediana, variação e desvio padrão obtidos a partir da análise dos 48 vídeos com os instrumentos de avaliação DISCERN e JAMA.

\begin{tabular}{|c|c|c|c|c|c|c|c|c|c|c|c|c|c|c|}
\hline & \multicolumn{10}{|c|}{ DISCERN } & \multicolumn{4}{|c|}{ JAMA } \\
\hline & 1 & 2 & 3 & 4 & 5 & 6 & 7 & 8 & 9 & 10 & 1 & 2 & 3 & 4 \\
\hline Média & 4,8 & 4,6 & 4,4 & 1,1 & 3,3 & 2,6 & 3,3 & 2,3 & 1,6 & 1,3 & 0,7 & 0,1 & 1,8 & 1 \\
\hline Mediana & 5 & 5 & 5 & 1 & 5 & 2 & 3 & 1 & 1 & 1 & 0 & 0 & 2 & 1 \\
\hline Variação & $1-5$ & $1-5$ & $1-5$ & $1-5$ & $1-5$ & $1-5$ & $1-5$ & $1-5$ & $1-5$ & $1-5$ & $0-2$ & $0-3$ & $0-3$ & $1-1$ \\
\hline Desvio padrão & 0,8 & 0,9 & 1 & 0,6 & 2 & 1,6 & 1,5 & 1,7 & 1,4 & 1 & 0,9 & 0,4 & 0,6 & 0 \\
\hline
\end{tabular}

Fonte: Próprios autores.

A Tabela 4 mostra a quantidade de vídeos e respectivas variações das notas obtidas pelos critérios de avaliação do DISCERN e do JAMA, divididos por quartis.

Tabela 4: Número de vídeos e as variações de suas notas, separados em quartis.

\begin{tabular}{ccc}
\hline & Número de vídeos (variação) & JAMA \\
\hline Quartis & DISCERN & $34(1-4)$ \\
Q1 (25\%) & $2(14-14)$ & $14(5-8)$ \\
Q2 (50\%) & $24(21-29)$ & 0 \\
Q3 (75\%) & $19(30-38)$ & 0 \\
Q4 (100\%) & $3(42-43)$ & 0 \\
\hline
\end{tabular}

Fonte: Próprios autores.

Segundo a média calculada a partir dos valores numéricos das classificações dos vídeos, as perguntas 4, 9 e 10 do DISCERN não obtiveram uma classificação satisfatória, ficando abaixo de 2. Com a somatória dos valores médios das notas de cada item foi obtido um valor de 29,5, sendo os vídeos considerados de qualidade média. A melhor nota obtida foi no vídeo de número 40, o qual obteve 43 pontos, sendo considerado de ótima qualidade visto que a nota máxima é 50 . O vídeo de número 9 e o 13 foram os vídeos analisados de pior qualidade e obtiveram 14 pontos, sendo considerado pelo DISCERN de péssima qualidade (variação de 14 a 43). Como mostra a tabela 4, a maioria dos vídeos (79,2\%) obteve uma nota maior que $50 \%$.

Foi possível verificar que alguns vídeos obtiveram uma média significativamente baixa no quesito das fontes de informação utilizadas, além do autor e produtor (item 4) e apenas um vídeo forneceu essa informação, recebendo a nota máxima (5). Os demais, como não forneceram a informação, nota mínima (1), a média aritmética entre todos os vídeos foi de 1,08. Outro quesito que não recebeu uma boa avaliação foi o item que avaliava se a informação de como as opções de tratamento afetam a qualidade de vida geral (item 9), sendo a média de 1,63, já que a maioria dos vídeos não forneciam este dado. Além desses, o último item do DISCERN adaptado, o décimo, no qual era avaliado se o vídeo fornecia suporte para decisão compartilhada, a média obtida foi de 1,33 .

A avaliação da confiabilidade dos vídeos realizada segundo os critérios propostos pelo JAMA mostrou que nenhum vídeo seria considerado confiável por apresentarem nota menor que 3. A média obtida a partir dos valores médios de cada item do JAMA avaliado foi de 0,89 . O vídeo com a pior avaliação obteve 0,25 de média e o melhor avaliado obteve 2 (variação de 0,25 a 2), portanto os vídeos foram considerados não confiáveis. Na tabela 4, 70,8\% dos vídeos tiveram uma nota menor que $25 \%$. Por fim, verificou-se que os vídeos avaliados apresentam deficiências que poderiam ser sanadas a partir da inclusão de informações que demonstrem sua qualidade e confiabilidade. 


\section{Discussão}

O aprendizado por meio de recursos midiáticos vem crescendo cada vez mais na área educativa, sendo o YouTube ${ }^{\circledR}$ uma plataforma comumente utilizada, principalmente devido ao livre acesso. Dessa forma, existe uma necessidade de análise da qualidade e confiabilidade dos conteúdos divulgados em vídeos educativos, em especial dos que tratam sobre assuntos relacionados à saúde e são direcionados ao público infantil. Muitos vídeos podem divulgar informações equivocadas e/ou desatualizadas, e por isso, a população pode estar sendo influenciada negativamente em suas condutas e de suas famílias. Em contrapartida, vídeos com informações adequadas e atualizadas podem ser utilizados como importante material de apoio para estudantes e para a população em geral (De Moraes Pinto, et al., 2015).

Foi constatado um alto número de visualizações e interações em alguns dos vídeos incluídos. Estes resultados corroboram com o número de visualizações encontrados em uma análise de vídeos educacionais sobre odontologia, realizada em 2011 na Universidade de Gotinga, na Alemanha, na qual foram encontrados vídeos que chegaram a 70 milhões de visualizações (Knosel, et al., 2011). Há uma tendência para um aumento no número de periódicos e instituições acadêmicas usando seus próprios canais no YouTube ${ }^{\circledR}$ para fins educacionais (Knosel, et al., 2011). Ademais, é possível que ocorra a visualização de um vídeo por várias vezes, principalmente pelo público infantil, podendo auxiliar o aprendizado e aumentar a assimilação de seu conteúdo, além de aumentar o número de visualizações.

Os vídeos podem, naturalmente, prender a atenção de um determinado público, o que é de importância para a divulgação de um conteúdo para um contingente maior de pessoas, das quais podem ter acesso à informação desejada por meio de uma busca simples no YouTube ${ }^{\circledR}$. Para o processo de aquisição de um conhecimento, há a necessidade da criação de uma representação interna de estimulação sensorial, vinculado a procedimentos de repetição e prática, atenção, profundidade de processamento, organização, formação de imagens e tipo de crenças cognitivas subjacentes (Da Costa Pinto, 2001). Isto mostra o potencial educativo desses meios de comunicação, cada dia mais utilizados. Consequentemente, é válida uma maior atenção na produção de vídeos, visando uma melhor qualidade de conteúdo de divulgação de informações adequadas e atualizadas.

$\mathrm{Na}$ Odontologia, a desinformação pode influenciar os pacientes a cuidados deficientes, levando a prejuízos à saúde bucal e consequentemente, levando a problemas sociais, estéticos, físicos e financeiros. Muitas vezes, o foco de vídeos sobre prevenção da cárie dentária pode estar centrado na venda de um produto ou marca, e acabar deixando o potencial educativo em segundo plano. Além disso, a falta de conhecimento da população sobre os cuidados com a saúde bucal e sua limitada capacidade de analisar criticamente as informações facilita a disseminação de conhecimentos errôneos (Maior, et al., 2015). Por isso, é necessário que produtores de vídeos apresentem suas fontes de consulta, para incentivar o público a buscar o conhecimento na literatura científica disponível. Em um estudo sobre saúde bucal em meios midiáticos, 10,8\% das informações analisadas estavam incorretas, sendo algumas delas fornecidas por profissionais dentistas (Maior, et al., 2015). Uma explicação para isso pode ser a desatualização dos profissionais, que muitas vezes não acompanham os avanços científico-tecnológicos, difundindo conceitos ultrapassados (Araújo, et al., 2007).

Foi possível verificar neste estudo que muitos dos vídeos cuja temática era a higienização dos dentes apresentaram uma quantidade excessiva de dentifrício na escova dental. A quantidade utilizada pelos personagens dos vídeos é comprovadamente contraindicada, pelos riscos de deglutição pela criança e consequente efeitos colaterais. Um dos compostos do creme dental é o fluoreto, que tem efeito comprovado no controle da cárie dentária, mas se ingerido de forma excessiva e prolongada pode levar à fluorose dentária, um distúrbio que interfere na formação do esmalte dentário (Fejerskov, et al., 1997). É claro que o uso de uma maior quantidade de creme dental no momento da escovação aumentará as vendas e consequentemente os lucros de empresas deste setor, entretanto, nem sempre a indústria e as empresas estão atentas aos cuidados necessários à saúde do consumidor, como percebe-se com a quantidade de dentifrício que tem sido utilizada nos vídeos sobre escovação. 
Assim, sugere-se que as Instituições de Ensino Superior (IES) auxiliem na divulgação do conhecimento científico e produção de conteúdos educacionais, baseados em evidências atualizadas, para serem compartilhados em plataformas de livre acesso, como o YouTube ${ }^{\circledR}$. Ainda as IES estão aquém de todo o potencial que essas plataformas oferecem aos seus estudantes de se tornarem autores, narradores, contadores de histórias e divulgadores, ficando atreladas aos métodos da educação clássica (Moran, 2013; Mattar, 2009).

Ao avaliar a qualidade dos vídeos, segundo o instrumento DISCERN, verificou-se uma variabilidade nas notas, com uma quantidade maior de vídeos com notas intermediárias e um número pequeno com notas muito boas e muito ruins. A nota média pelos critérios do DISCERN alcançou um valor acima da média, sendo as menores médias, dos itens 4, 9 e 10. Este resultado pode ser explicado pelo fato de os criadores dos vídeos não terem informado as referências utilizadas para a produção dos mesmos. Além disso, a maioria dos vídeos não informava como as opções de tratamento afetariam a qualidade de vida, e não forneciam suporte para a tomada de decisão compartilhada. A maioria dos vídeos ficou com uma nota acima da média, pois obtiveram boas notas em outros quesitos avaliados.

Avaliando os vídeos a partir dos critérios do instrumento do JAMA, nenhum vídeo obteve nota maior que 3 a partir dos quesitos analisados. Este resultado pode ser explicado pelos itens pobremente descritos sobre a autoria, fonte de informações, declaração de propriedade, publicidade e conflitos de interesses e a data da publicação. Desta forma, verificou-se que houve pouca preocupação em mostrar a confiabilidade das fontes de informações. É possível que os produtores dos vídeos possam ter utilizado fontes de informações confiáveis, entretanto esses não detalharam ou fizeram o registro nos vídeos ou em sua descrição.

A diferença nos resultados obtidos da utilização dos dois instrumentos de avaliação é explicada uma vez que o JAMA avalia a confiabilidade dos vídeos, sem levar em consideração o conteúdo apresentado, já o DISCERN sofreu adaptações para avaliar aspectos relevantes do conteúdo de um vídeo educativo sobre prevenção, por meio dos 10 quesitos apresentados neste trabalho. Da mesma forma, alguns trabalhos na literatura, como o de Ferhatoglu e colaboradores (2019), utilizaram o JAMA e o DISCERN como instrumentos de avaliação e obtiveram notas baixas em vídeos do YouTube ${ }^{\circledR}$, relacionados a distúrbios da coluna vertebral e doenças ortopédicas: a média geral do JAMA foi de 1,8 e 1,9 em cada quesito do DISCERN. Vale ressaltar que o YouTube ${ }^{\circledR}$ não foi criado com a finalidade de ser uma plataforma para o ensino na área da saúde, e, como tal, não possui características de revisão como as plataformas educacionais tradicionais. Essa é uma das explicações dos autores do trabalho, para a obtenção de médias tão baixas com os instrumentos de avaliação. Apesar disso, a plataforma tornou-se rapidamente uma das principais modalidades educacionais da atualidade (Ward, 2019). Certamente, o uso de instrumentos, como o JAMA e o DISCERN, por produtores de vídeos educativos, auxiliaria na produção de um conteúdo de melhor qualidade.

Baseado na necessidade da melhoria da confiabilidade, qualidade das informações e da produção de vídeos educativos para o público infantil, recomenda-se que: tenha uma boa qualidade de imagem e áudio; tenha uma história e linguagem que possa ser compreendida pelas crianças; tenha fontes de informações confiáveis, baseadas em evidências científicas atualizadas, sendos estas informadas no vídeo ou em sua descrição; tenha harmonia nas cores, harmonia nas músicas; passe o vídeos por um instrumento de avaliação, como o JAMA ou DISCERN, antes de sua publicação; sugira que o público busque a confirmação das informações na literatura; seja um vídeo que gere emoções, seja de humor, tristeza, gere um sentimento de empatia em quem o assiste, fazendo com que o público sinta os prejuízos de não executar a prevenção; traga a visão de mundo do público o qual serão destinados os vídeos; busque uma avaliação de um profissional da área ou de uma instituição de ensino superior antes da sua publicação, para que conste em sua descrição, dando mais credibilidade ao vídeo.

Acredita-se que vídeos chamativos, divertidos, comoventes ou com uma situação que a pessoa que o assiste se identifique, podem ser mais envolventes, compartilhando e gerando mais interações. Na literatura ainda são necessários futuros 
estudos para avaliar outros tipos de vídeos educativos na área da saúde bucal e compreender melhor o que faz as pessoas se interessarem por um vídeo e colocarem os aprendizados em prática.

\section{Conclusão}

Concluiu-se que os vídeos de animação para o público infantil analisados neste estudo, disponíveis no YouTube ${ }^{\circledR}$ sobre prevenção de cárie dentária variaram em termos de qualidade e de modo geral, foram considerados de baixa confiabilidade. Isso pode interferir no processo educativo de crianças e adultos para a prevenção de doenças, para a incorporação de hábitos saudáveis e para o autocuidado, uma vez que foram vistos por milhões de internautas. Fica evidente a importância dos cuidados para o aumento na qualidade e confiabilidade de vídeos educativos, uma vez que o ensino por meios digitais tem crescido de forma progressiva, sendo o principal método utilizado durante o período de isolamento social vivenciado no período da pandemia de COVID-19. Muitas vezes o público internauta geral e especialmente o infantil, não está preparado para buscar a veracidade e qualidade das informações. Assim, espera-se que as informações apresentadas nos vídeos, sejam baseadas em evidências científicas atualizadas, tendo suas fontes apresentadas e o conteúdo validado por profissionais qualificados.

\section{Agradecimentos}

Ao Conselho Nacional de Desenvolvimento Científico e Tecnológico (CNPq) pela concessão de Bolsa por meio do Programa Institucional de Bolsas de Iniciação Científica da Universidade Estadual de Maringá.

\section{Referências}

Aguiar, R. V., \& Cassiani, S. H. B. (2007). Desenvolvimento e avaliação de ambiente virtual de aprendizagem em curso profissionalizante de enfermagem. Revista Latinoamericana de Enfermagem, 15(6), 1086-91.

Aranha, E., \& Miranda, S. M. L. (2019). O papel dos influenciadores digitais no processo de intenção de compra. Novas Edições Acadêmicas.

Araújo, I. S., \& Cardoso, J. M. (2007). Comunicação e saúde. Editora Fiocruz.

Boctor, L. (2013). Active-learning strategies: The use of a game to reinforce learning in nursing education. A case study. Nurse education in practice, 13(2), 96-100.

Brasil (2016). Presidência da República. Secretaria Especial de Comunicação Social. Pesquisa Brasileira de Mídia 2016: Hábitos de consumo de mídia pela população brasileira. Brasília: Secom.

Brasil (2020). MEC. Portaria $\mathrm{n}^{\mathrm{o}}$ 544, de 16 de junho de 2020. Dispõe sobre a substituição das aulas presenciais por aulas em meios digitais, enquanto durar a situação de pandemia do novo conoravírus-Covid-19, e revoga as Portarias MEC nº 343, de 17 de março de 2020, nº 345, de 19 de março de 2020 e n 473, de 12 de maio de 2020 .

Burke, S. C., \& Snyder, S. L. (2008). YouTube: An Innovative Learning Resource for College Health Education Courses. International Electronic Journal of Health Education, 11, 39-46.

Charnock, D. (1998). The DISCERN handbook. Quality criteria for consumer health information on treatment choices. Radcliffe: University of Oxford and The British Library.

Conselho Regional de Medicina do Estado de São Paulo (CREMESP). Manual de princípios éticos para sites de medicina e saúde na Internet. http://www.cremesp.org.br/?siteAcao=PublicacoesConteudoSumario\&id=26

Creswell, J. W., \& Clark, V. L. P. (2013). Pesquisa de Métodos Mistos. Penso Editora.

Da Costa Pinto, A. (2001). Memória, cognição e educação: Implicações mútuas. Editora Edinova.

De Morais Pinto, A. P. C., et al. (2015). Análise de vídeos do youtube que abordam a técnica de cateterismo urinário de demora feminino. Cogitare Enfermagem, 20(2), 274-280.

Deslandes, S. F., \& Coutinho, T. (2020). O uso intensivo da internet por crianças e adolescentes no contexto da COVID-19 e os riscos para violências autoinflingidas. Ciência \& Saúde Coletiva, 25, 2479-2486.

Dinelli, W., Corona, S. A. M., Dinelli, T. C., \& Garcia, P. P. N. S. (2000). Desenvolvimento, aplicação e avaliação de um programa de orientação sobre higiene bucal junto a pré-escolares. Stoma, 13(57), 27-30. 
Fejerskov, O., Thylstrup, A., \& Larsen, M. J. (1977). Clinical and structural features and possible pathogenic mechanisms of dental fluorosis. European Journal of Oral Sciences, 85(7), 510-534.

Ferhatoglu, M. F., et al. (2019). Evaluation of the reliability, utility, and quality of the information in sleeve gastrectomy videos shared on open access video sharing platform YouTube. Obesity surgery, 29(5), 1477-1484.

Fujimaki, M. (2018). Orientando o paciente. Revista da Associação Paulista de Cirurgiões-Dentistas, 72(4): 678-679.

Gokcen, H. B., \& Gumussuyu, G. (2019). A quality analysis of disc herniation videos on YouTube. World neurosurgery, 124, e799-e804.

Henning, P. C. (1993). Internet@RNP.BR: um novo recurso de acesso à informação. Ciência da Informação, 22(1), 61-64.

Junior Fuculo, P. R., et al. (2015). Análise de vídeos do Youtube sobre prevenção de queimaduras. Revista Brasileira de Queimaduras, 14(2), 145-9.

Moura, G. B. F., \& Freitas, L. G. (2018). O Youtube como ferramenta de aprendizagem. REVELLI - Revista de Educação, Linguagem e Literatura, 10(3), 259-272.

Knösel, M., Jung, K., Bleckmann, A. (2011). YouTube, dentistry, and dental education. Journal of Dental Education, 75(12), 1558-1568.

Maior, G. M. S. C. S., \& Nyarwaya, R. N. (2015). Informações sobre saúde bucal, medicamentos e produtos odontológicos em mídia leiga. Trabalho de Conclusão de Curso, Universidade Federal do Rio Grande do Sul, Porto Alegre, RS, Brasil.

Mattar, J. (2009). YouTube na educação: o uso de vídeos em EaD. Universidade Anhembi Morumbi.

Monteiro, L. A. (2001). Internet como meio de comunicação possibilidades e limitações. Anais do Congresso Brasileiro de Ciências da Comunicação, Campo Grande, MS, Brasil, 24.

Moran, J. M., Behrens, M. A., Masetto, M. T. (2013). Novas Tecnologias e Mediação Pedagógica. Papirus Editora.

Petersen, P. E. (2003). The World Oral Health Report 2003: continuous improvement of oral health in the 21st century - the approach of the WHO Global Oral Health Programme. Community Dentistry and Oral Epidemiology, 31(1): 3-24.

Rodrigues, A., \& Silva, I.; Barros, R. (2017). Interações geradas a partir da visualização de vídeos em canais educacionais no YouTube. Internet Latent Corpus Journal, 7(2), 53-71.

Rodrigues, C. (2008). A presença do YouTube nos media - Razões e consequências. Anais do 5o Sopcom - Comunicação e Cidadania, Minho, Braga, Portugal.

Santos, P. A., Rodrigues, J. A., \& Garcia, P. P. N. S. (2003). Conhecimento sobre prevenção de cárie e doença periodontal e comportamento de higiene bucal de professores de ensino fundamental. Ciência Odontológica Brasileira, 6(1): 67-74.

Santos, K. T., Pacheco Filho, A. C., \& Garbin, C. A. S. (2012). Educação em saúde bucal na visão de acadêmicos de Odontologia. Arquivos em Odontologia, 48(2), 96-101.

Silberg, W. M., Lundberg, G. D., \& Musacchio, R. A. (1997). Assessing, controlling and assuring the quality of medical information on the Internet: Caveant lector et viewor-let the reader and viewer beware. JAMA, 277(15), 1244-1245.

Silva, N. K., Blumentritt, J. B., \& Cordeiro, F. R. Tecnologias Educacionais sobre Cuidados Paliativos no Instagram e no Youtube. Research, Society and Development, 10(7): 1-13. http://dx.doi.org/10.33448/rsd-v10i7.16534

Snelson, C. (2011). YouTube across the disciplines: A review of the literature. MERLOT Journal of Online Learning and Teaching, 7(1), 159-169.

Ward, M., et al. (2019). The educational quality of neurosurgical resources on YouTube. World neurosurgery, 130, 1-6. 